\title{
Human Papillomavirus Risk Perceptions Among Young Adult Sexual Minority Cisgender Women and Nonbinary Individuals Assigned Female at Birth
}

By Madina Agénor, Jaquelyn L. Jahn, Eriko Kay, Rachel A. Bishop, Sarah M. Peitzmeier, Jennifer Potter and S. Bryn Austin

CONTEXT: Some sexual minority women may be less likely than other women to engage in human papillomavirus (HPV) prevention behaviors. Although risk perceptions have been found to be associated with health behaviors, HPV risk perceptions among U.S. sexual minority women have not been examined.

METHODS: In 2016-2017, in-depth interviews were conducted in Boston with 29 sexual minority individuals aged 18-36 who were assigned female at birth (AFAB) and identified as women or nonbinary. Purposive sampling was used to recruit participants online, through community-based and student organizations, and by word of mouth.

This is the author manuscript accepted for publication and has undergone full peer review but has not been through the copyediting, typesetting, pagination and proofreading process, which may lead to differences between this version and the Version of Record. Please cite this article as doi: $10.1363 /$ psrh.12087

This article is protected by copyright. All rights reserved. 
Thematic analysis was employed to examine participants' HPV risk perceptions.

RESULTS: Participants incorrectly linked HPV risk to the exchange of genital fluids, and a hierarchy of perceived risk emerged in relation to sexual orientation: Individuals who engage in penile-vaginal sex with partners who were assigned male at birth (AMAB) were perceived to be at highest risk, and lesbians and individuals with only AFAB partners were perceived to be at low risk. Lesbians and participants with only AFAB partners identified sex with bisexual women or AFAB individuals with $\mathrm{AMAB}$ partners as a risk factor for HPV infection. Risk perceptions were shaped by health care providers' linking HPV risk to sex with AMAB individuals, a lack of discussion of HPV with parents and peers, and the exclusion of information on HPV and sexual minority women from school-based sex education.

CONCLUSION: Interventions providing sexual minority AFAB individuals with comprehensive, accurate and tailored information about HPV risk are needed.

Perspectives on Sexual and Reproductive Health, 2019, 51(1):TK, doi:TK 
Human papillomavirus (HPV) is the most common STD in the United States. ${ }^{1}$ Each year, approximately seven million U.S. women are newly infected with $\mathrm{HPV}^{2}{ }^{2}$ and about 23,300 develop a new cervical or other HPV-related cancer. ${ }^{3} \mathrm{HPV}$ can be acquired from sexual partners of any sex or gender through skin-to-skin sexual contact and the sharing of sex toys during vaginal, anal and oral sex. ${ }^{4}$ Sexual minority women (e.g., lesbians, bisexual and queer women, and women with same-sex sexual attractions or partners, regardless of sexual orientation identity), like non-sexual minority women, are therefore at risk of acquiring the infection. Indeed, a study using 2003-2012 National Health Interview Survey data showed that among U.S. women aged 20-59, the prevalence of HPV infection was 58\% among bisexual women, 36\% among lesbians and $41 \%$ among heterosexual women. Further, the prevalence was $57 \%$ among women who had had both male and female sexual partners, $34 \%$ among women reporting only female partners and $41 \%$ among those reporting only male partners. ${ }^{5}$

The HPV vaccine, approved for use in $2006,{ }^{17}$ is recommended for females aged 11-26. The vaccine effectively prevents HPV infection and, therefore, HPV-related diseases, including cervical cancer. ${ }^{1}$ Also, the correct and consistent use of barrier methods (e.g., condoms, dental dams) during sexual activity may provide some protection against HPV 
infection. ${ }^{6}$ However, prior research based on large national probability samples of U.S. women suggests that lesbians may be less likely than heterosexual women, and women with only female sexual partners may be less likely than women with only male sexual partners, to initiate HPV vaccination. ${ }^{7,8}$ Further, studies indicate that the majority of sexual minority women have never used a barrier method with a female sexual partner.9,10

Risk perceptions play an important role in shaping health behaviors. ${ }^{11}$ In a study of U.S. young adult women, Roberts et al. found that perceived HPV risk was positively associated with HPV vaccination. ${ }^{12}$ Thus, it is possible that perceptions of low HPV risk underlie the relatively lower prevalence of HPV vaccination initiation identified among lesbians compared with heterosexual women, and among women with only female sexual partners compared with women with only male sexual partners, in national probability samples.7,8 Moreover, Marrazzo et al. found that U.S. young adult lesbian and bisexual women generally believed that the risk of femaleto-female STD transmission was low, and that STD risk reduction behaviors were a concern primarily for heterosexual women. ${ }^{13}$ Thus, low levels of HPV risk perception may also contribute to reduced use of barrier methods, and hence increased risk of HPV infection among 
sexual minority women.

However, no study of which we are aware has examined HPV risk perceptions among sexual minority women in the United States. A qualitative U.S. study explored lesbian and bisexual young adult women's perceived risk of bacterial vaginosis and STDs in general, but not HPV in particular. ${ }^{13}$ In addition, a quantitative study investigated sexual orientation disparities in U.S. women's perceived risk of cervical cancer, but not HPV infection. ${ }^{14}$ Further, although we identified a mixed-methods study that specifically assessed lesbian and bisexual women's perceptions of HPV risk, the study was conducted in Australia and thus may not be generalizable to sexual minority women in the United States, given the two countries' differing social contexts, school-based sex education policies and HPV vaccination strategies. 15,16 Therefore, we designed a qualitative study to explore HPV risk perceptions and the possible influences on these perceptions among U.S. young adult sexual minority women. The present study may help inform programs and campaigns that facilitate access to accurate information about HPV risk and, in turn, promote HPV prevention among this population.

\section{METHODS}




\section{Sampling and Recruitment}

We used purposive sampling methods to select individuals who met the following eligibility criteria: Participants had to identify their sex as assigned female at birth (AFAB); their gender as woman; and their sexual orientation identity as lesbian, bisexual or queer.* They also had to be aged 18-36 (i.e., had been eligible for the HPV vaccine at some point in their lives) and live in the greater Boston area. We used maximum variation sampling to ensure the inclusion of individuals of diverse sexual orientation identity and age-groups. ${ }^{18,19}$ In addition, we used quota sampling based on the major racial and ethnic groups in the greater Boston area to enroll approximately equal numbers of white individuals and people of color (namely, black, Latinx, Asian and multiracial individuals).18,19 We recruited participants through community-based and student organizations, listservs and Facebook groups that serve lesbian, bisexual and queer women in the Boston area. ${ }^{20}$ We also posted recruitment ads on Craigslist and used snowball sampling, asking participants to inform potentially eligible individuals in their social networks about the study. ${ }^{18-20}$

\section{Data Collection}

We conducted in-depth interviews with 29 sexual minority cisgender 
women (i.e., AFAB individuals who identify as women) and nonbinary individuals (i.e., AFAB individuals who identify as neither men nor women) to elicit detailed information and generate new insights pertaining to their HPV risk and prevention perceptions, attitudes and experiences. ${ }^{21}$ Interviews were conducted in Boston from November 2016 to November 2017; interviewers were of diverse racial, ethnic and sexual orientation backgrounds. They used an interview guide, which was developed on the basis of the scientific literature and reviewed by experts in lesbian, gay, bisexual, transgender and queer (LGBTQ) health. ${ }^{22}$ The guide consisted of open-ended questions and probes pertaining to the following topics: sexual and reproductive health care experiences; sexual orientation disclosure; differential treatment in the health care system; HPV risk perceptions; HPV vaccination perceptions, attitudes and experiences; and communication regarding HPV risk and vaccination. After participants gave written informed consent, interviews were conducted in person in a private room in a university office building and lasted 40-80 minutes (mean length of time, 60 minutes); they were conducted in English and audio-recorded. Participants received a $\$ 25$ gift card for their time. All research activities were approved by the Office of Human Research Administration at Harvard Longwood Medical Area. 


\section{Analysis}

Interview audio-recordings were transcribed and entered into Dedoose (version 7) for analysis. Transcripts were analyzed using a thematic analysis approach informed by a critical realist theoretical position, which led us to examine participants' HPV risk perceptions as realities shaped by and interpreted in the context of their own lived experiences and in relation to sexism, heterosexism and racism in the health care system in particular and society in general. ${ }^{23}$ Analysis began with immersion in the data and codebook development and refinement. The initial codebook was developed collaboratively among research team members, and included deductive codes (based on research questions and the literature) and inductive codes (based on the first three transcripts). Two independent coders applied the codebook to four additional transcripts to test its fit to the data and ensure interrater reliability. Codes were merged, refined and discarded as needed, and coding discrepancies were discussed and resolved by consensus. The revised codebook was then applied to all interview transcripts. ${ }^{23}$

Because the present article provides a descriptive account of young adult sexual minority women's HPV risk perceptions, only text fragments coded with the thematic label "HPV risk perceptions" were 
included in our analysis. Subthemes were identified by examining relationships between relevant text fragments, both within and across transcripts. Analytic memo writing, research team discussions and feedback from LGBTQ health experts aided in the development and refinement of subthemes. Full transcripts were reviewed to ensure that all relevant text fragments had been included and that subthemes fit the totality of the data. ${ }^{23}$ In addition, transcripts were reviewed systematically to identify commonalities and differences in HPV risk perceptions across sexual orientation and racial or ethnic groups. ${ }^{24}$ Although individuals' perceptions and behaviors are influenced by a variety of social and demographic characteristics (e.g., race, ethnicity, socioeconomic position, age), we describe individuals in terms of their sexual orientation, as this played a particularly salient role in shaping participants' HPV risk perceptions.

\section{RESULTS}

\section{Sample Characteristics}

The majority of participants (21) were aged 18-26. All had at least some college education, and most (23) had private insurance. Although all participants identified as cisgender women at the time of screening, four identified as nonbinary at the time of the interview. Most 
identified as queer (23), and similar numbers identified as white (14) or as people of color (15-five black, one Latinx, seven Asian or Pacific Islander, and two biracial or multiracial individuals). Many participants reported having had cisgender women or cisgender men sexual partners (26 and 19, respectively). Of those who had initiated HPV vaccination (24), most (17) had received the vaccine before age 18. Additional information about participants' social, demographic and health care characteristics is provided in the Appendix (Supporting Information).

\section{Perceived HPV Risk Based on Sexual Orientation}

In general, participants believed that all sexually active AFAB individuals were at some risk of acquiring HPV, regardless of the sexual orientation identity of those individuals. Although a few participants espoused the view that sexually active AFAB individuals of different sexual orientations had the same risk of HPV infection, a hierarchy of perceived HPV risk based on sexual behavior and sexual orientation identity emerged from the data. While HPV risk perceptions varied in relation to participants' sexual orientation identity, they were similar across racial and ethnic groups.

- Sexual behavior. Most participants characterized vaginal-penile 
sexual intercourse with individuals who were assigned male at birth (AMAB) as conferring the highest level of HPV infection risk; indeed, AMAB individuals were often described as HPV carriers. For example, when asked who she perceived to be at highest risk of acquiring HPV, a queer cisgender woman responded: "Those who are having penisvagina sex are at higher risk, because the penis often carries [HPV]." A few participants linked their belief that sex with AMAB individuals conferred the highest level of HPV risk to the risk of pregnancy associated with unprotected vaginal-penile sex with these individuals. For instance, referring to her risk of acquiring HPV and other STDs, a queer and gay cisgender woman stated:

“Since I've stopped having sex with men, I just don't worry about it as much. I think partly that's [because] the fear of pregnancy is somehow tied in with the fear of [STDs], and so now that I'm not having sex with people who will make me pregnant, I'm like, 'Bases are covered. I'm OK."'

All participants recognized that HPV infection was possible in the context of sex among AFAB individuals. Most incorrectly linked this risk to the exchange of genital fluids during genital-genital and oralgenital contact. For example, a queer cisgender woman stated: "I think of $[\mathrm{HPV}]$ as being sexually transmitted by fluids in the genital area. 
And so, I think of lesbian sex that's genital to genital as being a way to transmit it." Similarly, when asked what sexual behaviors may increase HPV risk between cisgender women, a bisexual, queer nonbinary participant said: "I assume oral [sex]. Anything that involves exchange of fluids." Seven participants mentioned that sex toys may facilitate the transmission of STDs in general, or HPV in particular, in the context of sex among AFAB individuals, through the exchange of genital fluids. Only two participants identified digital-genital contact as a source of HPV risk.

Most participants believed that the likelihood of HPV transmission in the context of sex among AFAB individuals was low, much lower than that of male-to-female transmission. A queer cisgender woman stated: "I understand that I could still get an [STD] if I had sex with a woman or someone with a vagina..., but the risk seems a lot lower from that." Similarly, speaking about the range of sexual behaviors through which HPV can be transmitted, a queer and gay cisgender woman explained:

"Between women..., if someone was using their fingers, I think the only way you could transmit something was if you had a cut on your hand. Or [if] there was some kind of fluid being exchanged. And so, to me, [sex between women] seems inherently so much less [risky] than penis-in-vagina sex." 
Even when participants did not personally hold these beliefs, they noted that such beliefs were pervasive among their peers. For example, a queer cisgender woman noted:

“At least for cis women, I hear a misunderstanding that you can't get [HPV] if you're with other women. And I feel like you still can; I'm pretty sure. But it's like, 'Oh, well, somebody's not ejaculating in me.' Then I think it's fine."

While endorsing the belief that the risk of HPV transmission in the context of sex among AFAB individuals was generally low, several participants with only AFAB sexual partners identified having AFAB partners who have had $\mathrm{AMAB}$ partners as a notable risk factor. According to one such queer and gay cisgender woman:

"If someone has had a long sexual history with men, to me, that's a higher risk each time. Whereas even if someone has been with a lot of women, I don't associate [that with] as much of a chance of inheriting an [STD] in a sexual encounter between women."

Similarly, when asked whether she was concerned about HPV or cervical cancer, a queer cisgender woman responded: “I haven't been with a partner that's had sex with men..., so that hasn't been an issue."

Among participants with both $\mathrm{AFAB}$ and $\mathrm{AMAB}$ sexual partners, all but one perceived no difference in the risk of acquiring HPV based on 
sexual partners' sex or gender. For example, one of these participants, a bisexual and queer cisgender woman, stated: “It's not semen coming out that's giving you HPV. It's fluid-to-fluid contact, and a lot of women tend to have fluid-to-fluid contact."

- Sexual orientation identity. Several lesbian and gay participants believed that they were much less likely to acquire HPV than heterosexual and, especially, bisexual women. These participants attributed perceived differences in HPV risk across sexual orientation groups to presumed differences in the sexual behaviors of heterosexual and bisexual women and those of lesbians (i.e., having fewer sexual encounters or partners and not engaging in sex with cisgender men). Describing her likelihood of acquiring STDs, including HPV, a lesbian nonbinary individual said: "I'm going to be honest, in terms of [STDs], I just feel like maybe there's a thought like, 'I'm a lesbian, I'm probably not going to get as many-or be as exposed-as heterosexual or bisexual folks.'” When asked to elaborate, she explained: “I don't know, I feel like I would be having more frequent sex [if I weren't a lesbian]." Further, a queer and lesbian cisgender woman noted: "I would assume that someone who was bi, because they're having sex with men,... would be more at risk than me." Moreover, a gay cisgender woman stated: "If I have a new partner, I still want them to get tested- 
especially if that person identifies as bi." In contrast, all but one bisexual participant perceived no difference in the risk of acquiring HPV across sexual orientation groups. For example, a queer and bisexual cisgender woman said: "I haven't heard anything about HPV being related to sexual orientation."

\section{Sources of HPV Risk Perceptions}

Participants identified health care providers, parents, peers and schoolbased sex education as sources that influenced their HPV risk perceptions - through what they said and what they did not say about HPV infection risk among sexual minority women.

- Health care providers. Several participants described the important role that health care providers played in shaping their HPV risk perceptions. Specifically, providers influenced participants' perceptions by informing them that they were not at risk of acquiring HPV if they engaged in sex only with cisgender women. For example, a queer cisgender woman noted: "From what I understood in my conversation with my gynecologist, I'm not really at risk for [HPV] if I'm not sexually engaged with men." Similarly, a queer cisgender woman described how health care providers linked the risk of acquiring HPV and other STDs to the risk of pregnancy and thus to sex with AMAB 
individuals: "I feel like a lot of doctors will say, ‘Oh, you're good [because you have sex with only AFAB individuals]. You're not going to get pregnant. You're fine. Don't worry about these things.'”

In addition, participants described how providers influenced their perceptions by linking the need for Pap tests and HPV vaccines to sexual activity with cisgender men. For example, when asked if she had spoken with her health care provider about HPV, a lesbian cisgender woman responded: "A little bit. She asked me if I had gotten a Pap smear before, and I [said that] I hadn't because I've never been sexually active with a male. So, she said not to worry about it." Referring to a conversation with a provider about cervical cancer screening, a queer and lesbian cisgender woman shared:

"There was kind of this, 'well, the reason that you get Pap smears is around HPV or issues with your cervix. And since you're not having sex with men, your risk of HPV and cervical cancer is kind of low.' So, I got the sense that she was saying that I didn't need to come in for these regular checkups."

Further, regarding a conversation about HPV vaccination with the same provider, that participant added: "She had said, 'You could get [the HPV vaccine] in case you have sex with men at some point.'"

-Parents. Most participants reported rarely discussing HPV with their 
parents, which contributed to the perception that their risk of acquiring HPV was low or negligible. For example, referring to conversations about HPV risk and vaccination with her father, a queer cisgender woman noted: "He hasn't mentioned it to me, and I think in his mind, it's because I'm not involved with men." When asked if she had ever talked about HPV or STD prevention with her parents, a gay cisgender woman reported that she had not with either parent. She continued: "I think my sister is more likely to have had that conversation, because she identifies as straight." Even when participants described engaging in conversations about HPV or STD prevention with their parents, many reported being told that the risk of transmission in the context of sex among $\mathrm{AFAB}$ individuals was low. One queer and lesbian cisgender woman commented that she and her parent "talked... when [she] was young about dental dams, or Saran Wrap, or gloves" and "about being careful with fluids." However, she also noted that her parent informed her that, with "women, there is less risk."

- Peers. Several participants' perception of their HPV risk was shaped by the exclusion of HPV from conversations with their peers. For example, when asked if she ever talked about HPV with any of her friends, a gay cisgender woman responded: "Maybe briefly? Definitely less than other [STDs]. In general, my friend group is pretty focused on 
reproductive health and body positivity. We talk about those things a lot. But I don't think HPV comes up." When asked the same question, a bisexual and queer nonbinary AFAB participant stated: "Not really. It hasn't really been on my radar.... So, I guess if I did have a conversation, it would [have been] about cervical cancer, not HPV."

A few participants' HPV risk perceptions were shaped by their peers' STD prevention behaviors (or lack thereof). For example, a queer cisgender woman noted: "I think I asked one of my other queer friends if they ever use a dental dam and they were like, 'no.'” She later stated that this led her to believe that dental dams were not necessary and that STD transmission in the context of sex among AFAB individuals was low.

-School-based sex education. Some participants' perception that their risk of acquiring HPV was low or negligible was also shaped by the exclusion of information on HPV and sexual minority women from school-based sex education. Indeed, most participants reported that HPV was completely ignored in their sex education curriculum. A queer and pansexual cisgender woman noted: "I don't remember talking about HPV in sex ed in school. That's not something that gets discussed in the average sex ed class, I feel." Similarly, when asked if HPV-related content was covered in her sex education classes, a gay 
cisgender woman responded: "I don't think so. I think we talked about the standard-like, syphilis, gonorrhea, HIV. I don't think HPV was mentioned." Further, referring to the HPV vaccine, a queer woman noted: “I feel like there wasn't much conversation about it. I don't remember seeing flyers. Nobody in my high school talked about it. My public health class, from what I remember, was a joke, so I feel the dissemination of information did not happen. At all." However, exceptions included a lesbian cisgender woman who reported learning about HPV in a sex education class and a bisexual, queer nonbinary individual who had been told by a high school sex education teacher that the HPV vaccine was "useless."

Further, most participants observed that information on sexual minority women and discussion of STD transmission in the context of sex among AFAB individuals were also excluded from school-based sex education. According to a bisexual and pansexual cisgender woman: "A lot of the times, I feel like sex ed and safer sex practices are always taught to the heteronormative concept of sex." Similarly, a queer cisgender woman explained: “I think a big part of [people's HPV risk perceptions is formed by] the curriculum that happens at schools. It is very much geared towards cisgender, heteronormative culture." Heteronormative attitudes and the exclusion of sexual minority 
women's sexual experiences and concerns were also discussed by a bisexual, queer nonbinary participant in response to a question about what type of health information would be useful for sexual minority women to prevent HPV infection. This participant noted:

"So, making sex ed less heteronormative and including knowledge and talking about things like gloves and dental dams in middle and high schools would probably be huge to making those things not so obsolete, and not just... weird party favors available at the health center that you're never going to use because you don't know anybody else who uses them."

As a result of the focus on heteronormativity, several participants felt that STD prevention was not relevant to them. For example, a gay cisgender woman stated:

"At that time [in high school], I definitely knew I was gay, so I think I just felt like none of this applies to me. Now I know I still have to be worried about sexual health, but at the time, it felt like completely irrelevant information that doesn't apply.... I felt like there was nothing there for me."

\section{DISCUSSION}

To our knowledge, this is the first study to examine HPV risk 
perceptions among young adult sexual minority cisgender women and nonbinary AFAB individuals in the United States. We identified a hierarchy of perceived HPV risk based on both sexual behavior and sexual orientation identity. Specifically, the majority of participants perceived the risk of HPV infection to be highest among those who have penile-vaginal sex. In contrast, most participants incorrectly characterized the risk of acquiring HPV from AFAB sexual partners as low or negligible. Further, as found in other research conducted among U.S. young adult women, ${ }^{25}$ most participants erroneously attributed the risk of HPV transmission in the context of sex among AFAB individuals to the exchange of genital fluids and, in a few cases, to the risk of pregnancy, rather than to skin-to-skin sexual contact. These misperceptions may undermine individuals' engagement in HPV prevention strategies, including HPV vaccination and safer sex practices with AFAB sexual partners.

Our findings differ from those of a study by McNair et al., which showed that most Australian sexual minority women did not believe HPV could be transmitted between women. ${ }^{16}$ However, our results are aligned with the findings from another study by the same researchers that found that most sexual minority women perceived sex between women to be inherently safe because of the minimal transmission of 
bodily fluids. ${ }^{15}$ Other research suggests that individuals may derive their perceptions of HPV transmission from what they know about more typical STDs (e.g., HIV, chlamydia), which may be harder to transmit among sexual minority AFAB individuals, and are transmitted through bodily fluids, rather than through skin-to-skin contact. ${ }^{26}$ Our results are also congruent with McNair et al.'s finding that sexual minority women perceived sexual activity with bisexual women and women who have sex with cisgender men to be a source of HPV risk. ${ }^{15}$

Notably, our study contributes information on differences in HPV risk perceptions among sexual minority AFAB individuals by sexual orientation. Specifically, lesbian and gay participants and those with only AFAB partners tended to perceive themselves to be considerably less likely than bisexual women and those with AMAB sexual partners, respectively, to acquire HPV. In addition, these participants identified sex with bisexual women and $\mathrm{AFAB}$ individuals who have $\mathrm{AMAB}$ partners as a notable risk factor for HPV infection. Although the prevalence of such infection is relatively low among lesbians and women with only female sexual partners, individuals from all sexual orientation groups are at risk of acquiring HPV. ${ }^{5}$ We also found that, in our sample, participants' HPV risk perceptions did not vary by race or ethnicity. 
We identified health care providers as playing an important role in shaping HPV risk perceptions among young adult sexual minority AFAB individuals. This finding is similar to that of a study conducted among young adult women receiving care at Planned Parenthood and a hospital, which showed that the majority of participants who had heard of the HPV vaccine identified providers as an important source of information about HPV and HPV vaccination. ${ }^{25}$ However, in our study, several participants reported receiving erroneous messages from providers that engaging in sex with cisgender women conferred little to no risk of acquiring HPV. Other studies indicate that although health care providers can be a trusted source of HPV information, ${ }^{25}$ many lack the knowledge, attitudes and communication skills necessary to convey accurate, nonjudgmental and tailored information about HPV risk and prevention to sexual minority women. ${ }^{27-29}$ Indeed, education on LGBTQ health in general, and sexual minority women's health in particular, is lacking in both medical and nursing training programs, ${ }^{30,31}$ and providers often make erroneous assumptions about the sexual health needs of sexual minority women.27,28,32 Further, health care providers often do not receive training in sexual health, which may make discussions on these topics uncomfortable for them and undermine their ability to assess HPV risk and provide accurate HPV information 
to sexual minority women..$^{27,33}$ Moreover, providers often deliver services and information in the context of institutions and systems that assume and value heterosexuality, which further undermines sexual minority women's access to accurate and tailored sexual health information in health care settings. ${ }^{27,28}$

As in other studies, ${ }^{25,34}$ participants identified parents and peers as important influences on HPV risk perceptions. Indeed, several individuals reported that their perception that they were at low or negligible risk of HPV infection was influenced by the messages they did and did not receive from their parents about preventing HPV and other STDs. Research examining parental influences on the health of LGBTQ adolescents and young adults is scarce, and the results are mixed and primarily focused on young sexual minority men; however, some studies suggest that there may be an association between LGBTQ youths' sexual health and parental and parent-child relationship characteristics. ${ }^{35,36}$ In addition, other studies suggest that mothers may play an especially important role in communicating information about HPV risk and prevention in particular, and sex and sexual health in general, to female adolescents and young adults.12,37 Of note, Roberts et al. found that mother-daughter communication about sexual behaviors and STDs, including HPV, was positively associated with the perceived 
likelihood of acquiring HPV in a sample of U.S. women aged 18-25.12 Further, participants described how their HPV risk perceptions were influenced by a lack of peer-to-peer communication about HPV, as well as by their peers' STD prevention norms and practices. Although research on peer influence mirrors research on parental influence in terms of scope, results and focus, some studies indicate that communication about sexual health with peers (including regular sexual partners especially) and peer norms about safer sex practices may be associated with positive sexual health outcomes among LGBTQ adolescents and young adults. ${ }^{36}$

Moreover, several participants indicated that their HPV risk perceptions were shaped by the exclusion of information on both HPV and sexual minority women from school-based sex education. Indeed, sex education has historically focused on preventing pregnancy or HIV infection through abstinence or condom use in the context of penilevaginal intercourse; ${ }^{38-40}$ this focus may have fueled participants' perceptions that this sexual behavior confers the highest risk of HPV infection. Further, although 24 states and the District of Columbia mandated sex education in 2018, only nine states required that discussions of sexual orientation be inclusive of LGBTQ youth. ${ }^{40}$ The exclusion of LGBTQ sexual experiences and sexual health concerns 
from school-based sex education may have informed participants' perceptions that the risk of acquiring HPV in the context of sexual activity among AFAB individuals is low or negligible. Such perceptions may also be shaped by the exclusion of sexual minority women's specific and unique needs and concerns, including those regarding HPV risk and prevention, from other types of sexual health information (e.g., online materials). ${ }^{15,41}$ For example, in a 2012 review of online sexual health information, Lindley et al. identified only 25 unique and functioning websites pertaining to the sexual health concerns of lesbians. Of these, only 16 included information about HPV, and one discussed HPV vaccination. ${ }^{41}$

\section{Limitations}

Our findings should be interpreted in light of several limitations. First, all participants had at least some college education, and almost all were enrolled in a private health plan. Thus, our findings may not be applicable to women with less education, or women who are enrolled in a public health plan or lack insurance. Second, the majority of participants had received the HPV vaccine prior to age 18. Given their young age at vaccination, they were not able to describe in detail how their HPV risk perceptions prior to HPV vaccination had influenced 
whether they obtained the vaccine. Lastly, we were not able to assess whether perceived HPV risk varied by race or ethnicity. Thus, additional research with samples that include larger numbers of black, Latinx, Asian and Pacific Islander, multiracial and Native American individuals is needed to examine possible racial and ethnic differences in HPV risk perceptions among the sexual minority AFAB population, which may have been masked in the present study.

\section{Conclusions}

Our results have important implications for future research. Specifically, longitudinal qualitative and quantitative studies that assess HPV risk perceptions among adolescent and young adult sexual minority AFAB individuals who have not yet received the HPV vaccine are needed to assess whether and how perceived risk is associated with vaccination in this group. Moreover, research is needed to better understand how vaccination may influence HPV risk perceptions, as well as how perceptions are related to safer sex practices, among this population. Further, future studies should explore differences in HPV risk perceptions among this population in relation to relevant social factors other than sexual orientation, race and ethnicity, such as gender identity, gender expression and socioeconomic position. 
Our findings also have important implications for practice. They suggest that educational programs and campaigns (e.g., school-based, community-based, online) that provide sexual minority AFAB individuals with comprehensive and accurate information about their risk of acquiring HPV through skin-to-skin contact with sexual partners of any sex or gender from a nonheteronormative and affirming perspective are needed to address misperceptions about HPV risk, and may provide this population with the knowledge they need to make informed decisions about HPV prevention, including HPV vaccination and safer sex practices. ${ }^{38,39,41-43}$ Moreover, because health care providers were identified as an important influence on HPV risk perceptions, curricular and training interventions could help provide clinicians with accurate information about HPV risk among sexual minority AFAB individuals; challenge their misconceptions about HPV transmission in the context of sex among AFAB individuals; and enhance their nonjudgmental, nonheteronormative and patient-centered sexuality and sexual health communication skills. ${ }^{28,44,45}$

Also, interventions that provide parents and caregivers with the knowledge and communication skills they need to deliver accurate information about HPV risk and prevention to their adolescent and young adult sexual minority AFAB children may be beneficial..$^{35,36}$ In 
addition, interventions that facilitate peer-to-peer communication about HPV and target peer norms related to HPV transmission in the context of sex among AFAB individuals may help address misperceptions about HPV risk in this population. ${ }^{36}$ Along with improved providerand systems-level practices that ensure access to and delivery of respectful, patient-centered and high-quality care, $28,32,46$ promoting accurate HPV risk perceptions among sexual minority cisgender women and nonbinary AFAB individuals may ultimately contribute to the prevention of cervical cancer and other HPV-related diseases in this population. 
FOOTNOTE

FN A

*To be eligible for the study, individuals had to identify as lesbian, bisexual or queer, but could also identify with other sexual orientations (e.g., gay, queer, pansexual).

This article is protected by copyright. All rights reserved. 


\section{REFERENCES}

1. Dunne EF et al., CDC grand rounds: reducing the burden of HPV-associated cancer and disease, Morbidity and Mortality Weekly Report, 2014, Vol. 63, No. RR-4.

2. Satterwhite CL et al., Sexually transmitted infections among U.S. women and men: prevalence and incidence estimates, 2008, Sexually Transmitted Diseases, 2013, 40(3):187193, doi:10.1097/OLQ.0b013e318286bb53.

3. Jemal A et al., Annual report to the nation on the status of cancer, 1975-2009, featuring the burden and trends in human papillomavirus(HPV)-associated cancers and HPV vaccination coverage levels, Journal of the National Cancer Institute, 2013, 105(3):175-201, doi:10.1093/jnci/djs491.

4. Marrazzo JM and Gorgos LM, Emerging sexual health issues among women who have sex with women, Current Infectious Disease Reports, 2012, 14(2):204-211, doi:0.1007/s11908012-0244-x.

5. Reiter P and McRee A, HPV infection among sexual minority women: Does it matter how sexual orientation is measured? Cancer Epidemiology, Biomarkers and Prevention, 2016, 25(3):559-560, doi:10.1158/1055-9965.EPI-16-0085.

6. National Cancer Institute, HPV and cancer, 2015, http://www.cancer.gov/aboutcancer/causes-prevention/risk/infectious-agents/hpv-fact-sheet.

7. Agénor $\mathrm{M}$ et al., Sex of sexual partners and human papillomavirus vaccination among U.S. girls and women, American Journal of Preventive Medicine, 2016, 50(3):318-327, doi:10.1016/j.amepre.2015.08.025.

8. Agénor M et al., Sexual orientation identity disparities in awareness and initiation of the human papillomavirus vaccine among U.S. women and girls: a national survey, Annals of Internal Medicine, 2015, 163(2):99-106, doi:10.7326/M14-2108. 
9. McCune KC, Imborek KL and Stockdale CK, Sexual preventative health in U.S. sexual minority women: a review, Proceedings in Obstetrics and Gynecology, 2017, 7(1):1-16, doi:10.17077/2154-4751.1329.

10. Schick V et al., Sexual behaviours, sex toy and sexual safety methods reported by women who have sex with women and men, Sexual Health, 2015, 12(4):287-299, doi:10.1071/SH14045.

11. Brewer NT et al., Meta-analysis of the relationship between risk perception and health behavior: the example of vaccination, Health Psychology, 2007, 26(2):136-145, doi:10.1037/0278-6133.26.2.136.

12. Roberts ME et al., Mother-daughter communication and human papillomavirus vaccine uptake by college students, Pediatrics, 2010, 125(5):982-989, doi:10.1542/peds.20092888.

13. Marrazzo JM, Coffey $\mathrm{P}$ and Bingham A, Sexual practices, risk perception and knowledge of sexually transmitted disease risk among lesbian and bisexual women, Perspectives on Sexual and Reproductive Health, 2005, 37(1):6-12, doi:10.1363/3700605.

14. Price JH et al., Perceptions of cervical cancer and Pap smear screening behavior by women's sexual orientation, Journal of Community Health, 1996, 21(2):89-105, doi:10.1007/BF01682301.

15. Power J, McNair R and Carr S, Absent sexual scripts: lesbian and bisexual women's knowledge, attitudes and action regarding safer sex and sexual health information, Culture, Health \& Sexuality, 2009, 11(1):67-81, doi:10.1080/13691050802541674.

16. McNair R, Power J and Carr S, Comparing knowledge and perceived risk related to the human papilloma virus among Australian women of diverse sexual orientations, Australian and New Zealand Journal of Public Health, 2009, 33(1):87-93, doi:10.1111/j.1753- 
6405.2009. 00345.x.

17. Markowitz LE et al., Quadrivalent human papillomavirus vaccine: recommendations of the Advisory Committee on Immunization Practices (ACIP), Morbidity and Mortality Weekly Report, 2007, Vol. 56, No. RR-2.

18. Patton M, Qualitative Evaluation and Research Methods, third ed., Thousand Oaks, CA: SAGE Publications, 2002.

19. Marshall MN, Sampling for qualitative research, Family Practice, 1996, 13(6):522-525, doi:10.1093/fampra/13.6.522.

20. Arcury T and Quandt S, Participant recruitment for qualitative research: a site-based approach to community research in complex societies, Human Organization, 1999, 58(2):128-133, doi:10.17730/humo.58.2.t5g838w7u1761868.

21. Tolley EE et al., Qualitative Methods in Public Health: A Field Guide for Applied Research, second ed., San Francisco: Wiley, 2016.

22. King N and Horrocks C, Interviews in Qualitative Research, London: SAGE Publications, 2010.

23. Braun V and Clarke V, Using thematic analysis in psychology, Qualitative Research in Psychology, 2006, 3(2):77-101, doi:10.1191/1478088706qp063oa.

24. Miles MB, Huberman $\mathrm{M}$ and Saldaña J, Qualitative Data Analysis: A Methods Sourcebook, third ed., Thousand Oaks, CA: SAGE Publications, 2014.

25. Unger $Z$ et al., Knowledge of HPV and HPV vaccine among women ages 19 to 26 , Women's Health Issues, 2015, 25(5):458-462, doi:10.1016/j.whi.2015.06.003.

26. Reyna VF and Adam MB, Fuzzy-trace theory, risk communication and product labeling in sexually transmitted diseases, Risk Analysis, 2003, 23(2):325-342, 
doi:10.1111/1539-6924. 00332.

27. McNair R, Risks and prevention of sexually transmissible infections among women who have sex with women, Sexual Health, 2005, 2(4):209-217, doi:10.1071/SH04046.

28. Hutchinson MK, Thompson AC and Cederbaum JA, Multisystem factors contributing to disparities in preventive health care among lesbian women, Journal of Obstetric, Gynecologic and Neonatal Nursing, 2006, 35(3):393-402, doi:10.1111/j.15526909.2006.00054.x.

29. Bonvicini KA and Perlin MJ, The same but different: clinician-patient communication with gay and lesbian patients, Patient Education and Counseling, 2003, 51(2):115-122, doi:10.1016/S0738-3991(02)00189-1.

30. Lim F, Johnson M and Eliason M, A national survey of faculty knowledge, experience and readiness for teaching lesbian, gay, bisexual and transgender health in baccalaureate nursing programs, Nursing Education Perspectives, 2015, 36(3):144-152, doi:10.5480/141355.

31. Amato $\mathrm{P}$ and Morton D, Lesbian health education: a survey of obstetrics and gynecology residency training programs, Journal of the Gay and Lesbian Medical Association, 2002, 6(2):47-51, doi:10.1023/A:1021941405832.

32. McNair RP, Lesbian health inequalities: a cultural minority issue for health professionals, Medical Journal of Australia, 2003, 178(12):643-645, https://www.mja.com.au/ journal/2003/178/12/lesbian-health-inequalities-cultural-minority-issue-healthprofessionals.

33. Apaydin $\mathrm{KZ}$ et al., Facilitators of and barriers to HPV vaccination among sexual and gender minority patients at a Boston community health center, Vaccine, 2018, 36(26):38683875, doi:10.1016/j.vaccine.2018.02.043. 
34. Gerend MA and Magloire ZF, Awareness, knowledge and beliefs about human papillomavirus in a racially diverse sample of young adults, Journal of Adolescent Health, 2008, 42(3):237-242, doi:10.1016/j.jadohealth.2007.08.022.

35. Bouris A et al., A systematic review of parental influences on the health and wellbeing of lesbian, gay and bisexual youth: time for a new public health research and practice agenda, Journal of Primary Prevention, 2010, 31(5-6):273-309, doi:0.1007/s10935010-0229-1.

36. Johns $\mathrm{MM}$ et al., Systematic mapping of relationship-level protective factors and sexual health outcomes among sexual minority youth: the role of peers, parents, partners and providers, LGBT Health, 2018, 5(1):6-32, doi:10.1089/lgbt.2017.0053.

37. McRee AL et al., Mother-daughter communication about HPV vaccine, Journal of Adolescent Health, 2011, 48(3):314-317, doi:10.1016/j.jadohealth.2010.07.006.

38. Elia JP and Eliason M, Discourses of exclusion: sexuality education's silencing of sexual others, Journal of LGBT Youth, 2010, 7(1):29-48, doi:10.1080/19361650903507791.

39. Gowen LK and Winges-Yanez N, Lesbian, gay, bisexual, transgender, queer and questioning youths' perspectives of inclusive school-based sexuality education, Journal of Sex Research, 2014, 51(7):788-800, doi:10.1080/00224499.2013.806648.

40. Guttmacher Institute, Sex and HIV education, State Laws and Policies (as of October 1, 2018), 2018, https://www.guttmacher.org/state-policy/explore/sex-and-hiv-education? gclid=EAIaIQobChMIqrePhvKM2wIVVySBCh38JAOSEAAYASABEgIp0_D_BwE.

41. Lindley LL, Friedman DB and Struble C, Becoming visible: assessing the availability of online sexual health information for lesbians, Health Promotion Practice, 2012, 13(4):472480, doi:10.1177/1524839910390314.

42. Lambert EC, College students' knowledge of human papillomavirus and effectiveness 
of a brief educational intervention, Journal of the American Board of Family Practice, 2001, 14(3):178-183, https://www.jabfm.org/content/14/3/178.full.pdf.

43. Wetzel C et al., Development of an HPV educational protocol for adolescents, Journal of Pediatric and Adolescent Gynecology, 2007, 20(5):281-287, doi:10.1016/j.jpag.2006.12.002.

44. Marrazzo JM, Barriers to infectious disease care among lesbians, Emerging Infectious Diseases, 2004, 10(11):1974-1978, doi:10.3201/eid1011.040467.

45. Levinson W, Lesser CS and Epstein RM, Developing physician communication skills for patient-centered care, Health Affairs, 2010, 29(7):1310-1318, doi:10.1377/hlthaff.2009. 0450.

46. Mayer $\mathrm{KH}$ et al., Sexual and gender minority health: what we know and what needs to be done, American Journal of Public Health, 2008, 98(6):989-995, doi:10.2105/AJPH.2007. 127811. 


\section{Authors' bios}

Madina Agénor is Gerald R. Gill Assistant Professor of Race, Culture and Society, Department of Community Health, Tufts University, Medford, MA. Jaquelyn L. Jahn is a Ph.D. candidate, Eriko Kay is research coordinator, Rachel A. Bishop is research assistant and S. Bryn Austin is professor-all in the Department of Social and Behavioral Sciences, Harvard T.H. Chan School of Public Health, Boston. Sarah M. Peitzmeier is research fellow, Department of Health Behavior and Biological Sciences, University of Michigan School of Nursing, Ann Arbor. Jennifer Potter is professor, Department of Medicine, Harvard Medical School, Boston.

\section{Acknowledgments}

This study was funded by grant 128863-PF-15-149-01-CPHPS from the American Cancer Society. The authors thank the study participants for their time and expertise, the community-based and student organizations for their assistance with participant recruitment, and the Sexual Orientation and Gender Identity and Expression Working Group at Harvard T.H. Chan School of Public Health for their feedback on preliminary results of the study.

Author contact: madina.agenor@tufts.edu 